\title{
An Adaptive Distance-Based Location Update Algorithm for PCS Networks
}

\author{
Vincent W.-S. Wong and Victor C. M. Leung \\ Department of Electrical and Computer Engineering \\ University of British Columbia \\ 2356 Main Mall, Vancouver, BC, Canada V6T 1Z4 \\ e-mail: vincentw@ece.ubc.ca, vleung@ece.ubc.ca
}

\begin{abstract}
In this paper, we propose a stochastic model to compute the optimal update boundary for the distance-based location update algorithm. The proposed model captures some of the real characteristics in the wireless cellular environment. The model can adapt to arbitrary cell topologies in which the number of neighboring base stations at different locations may vary. The cell residence time can follow general distributions which captures the fact that the mobile user may spend more time at certain locations than others. The model also incorporates the concept of a trip in which the mobile user may follow a particular path to a destination. For implementation, the decision of location update can be made by a simple table lookup. Numerical results indicate that the proposed model provides a more accurate update boundary in real environment than that derived from a hexagonal cell configuration with random walk movement pattern. The proposed model allows the network to maintain a better balance between the processing incurred due to location update and the radio bandwidth utilized for paging between call arrivals.
\end{abstract}

\section{INTRODUCTION}

In recent years, there has been a significant increase in the number of Personal Communications Service (PCS) subscribers around the world. One of the issues in mobility management is to track the location of the users [1]. Since mobile users are free to move within the coverage area, the network can only maintain the approximate location of each user. When a connection needs to be established to a particular user, the network has to determine the user's exact location within the cell granularity. The operation of the mobile terminal informing the network about its current location is known as location update, and the operation of the network determining the exact location of the mobile user is called terminal paging or searching.

Location update algorithms can be divided into two main groups: static and dynamic. In a static algorithm, location update is triggered based on the topology of the network. Examples include the conventional location area (LA) based scheme used in GSM systems. In a dynamic algorithm, location update is based on the user's call and mobility patterns. Examples include the distance-based, timer-based, and the movement-based schemes. In the distance-based scheme [2][3], a mobile terminal transmits an update signal whenever its distance (in terms of the number of cells) exceeds a certain threshold. In the timer-based scheme [4], a mobile terminal updates its location periodically. In the movement-based scheme [5], location update is performed when the number of boundary crossings between cells exceeds a certain value.

A number of novel location update algorithms have been

This work was supported in part by the Natural Sciences and Engineering Research Council of Canada under a Postgraduate Scholarship and Grant No. OGP0044286; scholarships from the Communications Research Centre, Industry Canada, and the University of British Columbia. proposed recently. In the predictive distance-based update scheme [6], the mobile terminal reports both its location and velocity during the update process. The network used this information to predict the mobile's location in future time. In [7], a state-based update scheme is analyzed where the system state includes the current location and the time elapsed since the last update. The LeZi update algorithm proposed in [8] can be considered to be a path-based update scheme in which the movement history rather than the current location is sent in an update message. In [9], the concepts of boundary location area and boundary location register were proposed to facilitate roaming between different wireless networks. The update distance is a function of the QoS factor, velocity ratio, and predefined threshold.

Although various location update algorithms have been proposed in the literature, the evaluations of these algorithms are often performed under certain unrealistic assumptions:

1. Structured cell configurations: Mesh or hexagonal cell configurations are used in two-dimensional models [3][5], and a linear model is used in the one-dimensional case [2][6]. Although these cell topologies simplify the analyses, they do not give an accurate representation of a realistic cellular network topology where the number of neighboring cells varies from cell to cell.

2. Exponential cell residence time distribution: Most of the work assumed the cell residence time follows a geometric (or exponential) distribution [2][6][10], which is assumed to be independent and identically distributed (i.i.d.) for all cells. The drawback of this assumption is that it does not capture an accurate representation of individual user mobility patterns, where a user may stay at certain locations (e.g., his home or office) for a relatively long period of time.

3. Symmetric random walk movement pattern: This assumption is commonly used to characterize individual movement behavior [2][3][5]. Although the random walk model simplifies the analysis, the direction of the mobile user is not taken into account. In general, a mobile user may travel with a destination in mind. Thus, the mobile's location in the future is likely to be correlated with its movement history.

In this paper, we focus on determining the update boundary for the distance-based scheme. Our goal is to develop an analytical model which can eliminate some of the unrealistic assumptions commonly used. We formulate the location tracking problem as a semi-Markov decision process [11]. There is a cost function associated with location update and another cost function associated with terminal paging. The objective is to determine the optimal update boundary so as to minimize the expected total cost between call arrivals. Distinct features of our model include [12]:

1. Applicable to arbitrary cell topologies: This feature captures the fact that the number of neighboring base stations at different locations may vary in real life. Some 
base stations may only have two neighboring base stations while others can have as many as six. Thus, our model is not restricted to structured cell configuration such as mesh or hexagonal.

2. Cell residence time can follow general distributions: This captures the fact that a mobile user may spend more time at certain cell locations (e.g., home or office) than other locations. In addition, various distributions can be used to model different cell sizes (e.g., macrocell, microcell, or picocell). The average residence time in each cell can be different.

3. Incorporate the movement history: The probability that the mobile user moves to a particular neighboring cell can depend on the location of the current cell or a list of cells recently visited. This movement pattern can incorporate the concept of a trip in which the user may choose a particular path to a destination.

The structure of this paper is as follows: In Section II, we introduce the notations and describe the model formulation. In Section III, we analyze the distance-based algorithm under a given cell residence time distribution and describe its implementation in arbitrary cell topology. Numerical results are presented in Section IV. Conclusions are given in Section V.

\section{MODEL FORMULATION}

In this section, we describe how to formulate the location tracking problem as a semi-Markov decision process. A Markov decision process model consists of five elements: decision epochs, states, actions, transition probabilities, and costs. In our model, the decision epochs correspond to the time instants when the mobile terminal moves from one cell to another (i.e., crossing the cell boundary).

When the mobile terminal crosses the cell boundary, it has to decide whether to update its location or not. We let the action set $A=\{0,1\}$, where " 1 " represents the action of performing location update and " 0 " represents the null action of no intervention. The mobile terminal chooses the action based on its current state information. For the model formulation, we let the state $s=(i, j)$, where $i$ represents the identifier of the current cell that the mobile terminal is residing, and $j$ represents the identifier of the cell in which the mobile terminal performed its last update.

Two cost functions are introduced to account for the network resources used for location update and terminal paging. The location update cost is assumed to be fixed and is denoted by $C_{U U}$. It reflects the update processing incurred on the database. The paging cost function is given by $h(i, j)$. The paging cost reflects the consumption of radio bandwidth utilized in determining the exact location of the mobile terminal. The paging cost is a function of the number of cells being paged and the number of search iterations performed. In this paper, we assume that the paging strategy follows the shortestdistance-first order. That is, when a paging event occurs, the search is conducted first at the user's last reported cell. If it is not found there, then the search is conducted in the next ring of cells in increasing distance order from the last reported cell, until the user is located. The maximum paging delay corresponds to the maximum number of search iterations allowed. At each search iteration, a set of cells are being paged simultaneously. This paging strategy has also been used in conjunction with other location update algorithms (e.g., [2][3][6]).

For a particular mobile user, different cell residence time distributions can be assigned to different cell locations. The average residence time in each cell can be different. This is achieved by letting $G(t \mid i)$ denote the cumulative distribution function of the cell residence time, given the identifier of the current cell is $i$. This captures the fact that a mobile user may spend more time at certain cell locations than the others. Different cell sizes (e.g., macrocell, microcell, picocell) can have different cell residence time distributions. Thus, the usual i.i.d. exponential cell residence time assumption can be relaxed. In this paper, we use $G(d t \mid i)$ to represent the timedifferential. That is, $G(d t \mid i)=d G(t \mid i)$.

For the movement pattern in an arbitrary cell topology, we let $P(k \mid i)$ denote the probability that the mobile user will move to neighboring cell $k$ when it leaves cell $i$. This captures the correlations of the user movement between two neighboring cells. Note that correlations between the directions of successive moves of the mobile user can also be incorporated if the state includes a history of the cells visited. Thus, the model can incorporate the concept of a trip in which the mobile user may follow a particular path to a destination. In addition, the above formulation does not assume any particular cell configurations. Therefore, the proposed model can adapt to arbitrary cell topologies.

\section{Distance-BASED UPDATE ALGORITHM}

\section{A Optimality Equations}

Since the network must track the user's location perfectly during a call, the user's location is known to the network when a call terminates. Thus, the time interval requiring mobility tracking is between the termination of the last call and the arrival of the next one. The total cost for location update and paging is calculated within this time interval. Let $v(i, j)$ denote the minimum expected total cost between call arrivals given state $(i, j)$. If the time between call arrivals is exponentially distributed with rate $\lambda$, then the optimality equations are given by:

$$
\begin{aligned}
v(i, j)= & \min \left\{\int_{0}^{\infty}\left(1-e^{-\lambda t}\right) h(i, j) G(d t \mid i)\right. \\
+ & \sum_{k}\left[\int_{0}^{\infty} e^{-\lambda t} v(k, j) P(k \mid i) G(d t \mid i)\right], \\
& C_{L U}+\int_{0}^{\infty}\left(1-e^{-\lambda t}\right) h(i, i) G(d t \mid i) \\
+ & \left.\sum_{k}\left[\int_{0}^{\infty} e^{-\lambda t} v(k, i) P(k \mid i) G(d t \mid i)\right]\right\}
\end{aligned}
$$

where $1 \leq i, j \leq N$ and $N$ denotes the number of base stations within the coverage area. Due to space limitations, please refer to [12] for the proof of the optimality equations. The first term in (1) denotes the expected total cost if no update is performed at state $(i, j)$, while the second term denotes the expected total cost if location update is performed at state $(i, j)$.

\section{$B$ Value Iteration Algorithm}

There are a number of algorithms available to solve equation (1). Examples include the value iteration or policy iteration algorithms [11]. The following value iteration algorithm finds a stationary deterministic optimal policy and the corresponding expected total cost. The solutions of the optimality equations corresponds to the minimum expected total cost $v(i, j)$ and the optimal policy $\delta(i, j)$. Note that the optimal policy $\delta^{*}(i, j)$ indicates the decision whether to update its location or not at state $(i, j)$. 
Algorithm

1. Set $v^{0}(i, j)=0$ for each state $(i, j)$ where $1 \leq i, j \leq N$. Specify $\varepsilon>0$ and set $n=0$.

2. For each state $(i, j)$ where $1 \leq i, j \leq N$, compute $v^{n}(i, j)$ by

$$
\begin{aligned}
v^{n+1}(i, j)= & \min \left\{\int_{0}^{\infty}\left(1-e^{-\lambda t}\right) h(i, j) G(d t \mid i)\right. \\
+ & \sum_{k}\left[\int_{0}^{\infty} e^{-\lambda t} v^{n}(k, j) P(k \mid i) G(d t \mid i)\right], \\
& C_{L U}+\int_{0}^{\infty}\left(1-e^{-\lambda t}\right) h(i, i) G(d t \mid i) \\
+ & \left.\sum_{k}\left[\int_{0}^{\infty} e^{-\lambda t} v^{n}(k, i) P(k \mid i) G(d t \mid i)\right]\right\}
\end{aligned}
$$

3. If $\left\|v^{n+1}-v^{n}\right\|<\varepsilon$, go to step 4. Otherwise increment $n$ by 1 and return to Step 2 .

4. For each state $(i, j)$ where $1 \leq i, j \leq N$, the stationary optimal policy $\delta(i, j)=0$ if

$$
\int_{0}^{\infty}\left(1-e^{-\lambda t}\right) h(i, j) G(d t \mid i)+\sum_{k}\left[\int_{0}^{\infty} e^{-\lambda t} v^{n}(k, j) P(k \mid i) G(d t \mid i)\right]
$$

$<C_{L U}+\int_{0}^{\infty}\left(1-e^{-\lambda t}\right) h(i, i) G(d t \mid i)$

$+\sum_{k}\left[\int_{0}^{\infty} e^{-\lambda t} v^{n}(k, i) P(k \mid i) G(d t \mid i)\right]$.

Otherwise, $\delta^{*}(i, j)=1$.

5. Stop.

There are a number of definitions for the function norm $\|$. $\|$. In this paper, the function norm is defined as $\|v\|=\max v(i, j)$ for $1 \leq i, j \leq N$. Convergence of the value iteration algorithm is ensured since the operation in Step 2 corresponds to a contraction mapping. Thus, the function $v^{\prime \prime}(i, j)$ converges in norm to $v(i, j)$.

\section{$C$ Implementation Considerations}

Having identified the different parameters involved in the model, we are now in a position to explain the steps that need to be taken in order to implement the model. First of all, the network controller has to assign cost functions for location update and terminal paging. It also has to maintain the mobility profile of each mobile user (i.e., its movement history and call history). Based on these information, the average residence time in each cell as well as the average call arrival rate can be estimated [13].

Given the input parameters (i.e., cost functions and various distributions), the value iteration algorithm can be used to calculate the optimal policy $\delta(i, j)$. Based on the optimal policy, the optimal update boundary can be determined. The optimal update boundary is then stored in a database (e.g., location register). After each location update or a call termination, the mobile terminal needs to download the list of the update boundary cell identifiers that corresponds to its current location. Whenever the mobile terminal moves to another cell, it compares the new cell identifier with the list of the update boundary cell identifiers. Location update is performed if the new cell is one of those update boundary cells.

The optimal update boundary stored in the location register needs to be revised whenever there is a change of the movement history, call history, or network topology (e.g.,
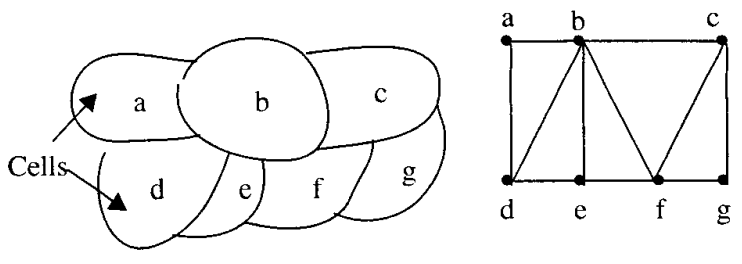

(a) The cell topology.

(b) Graph model showing the interconnections of the cells.

Figure 1 Cellular network topology represented by a graph model.

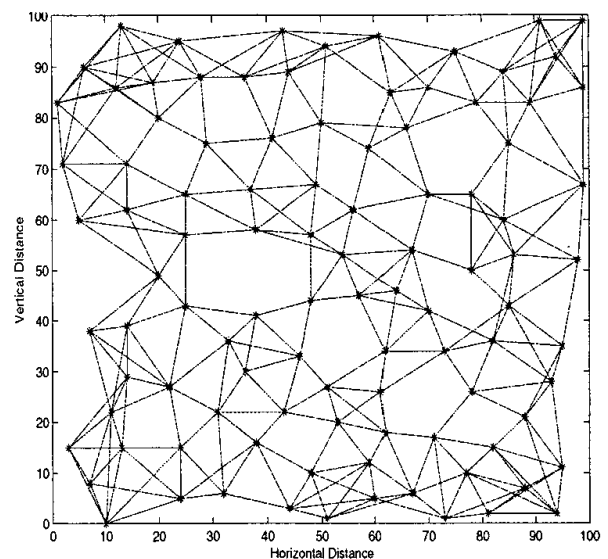

Figure 2 The nodes represent the location of the base stations. An edge between two nodes represents those two base stations are neighbors to each other.

installation of new base stations). The calculation of the optimal update boundaries can be performed off-line, e.g., whenever spare processing capacity is available at the network controller.

\section{NUMERICAL RESULTS AND DISCUSSIONS}

\section{A Simulation Assumptions and Specifications}

In our simulation environment, a graph model is used to represent the topology of a cellular network. In general, the interconnection of the cells can be modeled as a connected graph $G=(N, E)$, where the node set $N$ represents the set of cell or base station identifiers and the edge set $E$ represents the connectivity between two neighboring cells. For example, referring to Figure 1 , the node set $N=\{a, b, c, d, e, f, g\}$ and the edge set $E=\{(a, b),(a, d),(b, c), \ldots,(f, g)\}$.

In particular, we use a random graph model to represent the topology of a cellular network. The rationale behind using a random graph model is that: (1) the number of neighboring base stations for each base station can be different; and (2) only the nodes that are close together are connected. This models the connectivities of the neighboring base stations. The procedures of generating random graphs can be found in [14]. In our model, we consider a coverage area that consists of 100 base stations with an average node degree of 6 . An example of a random graph model is shown in Figure 2.

Since the mobile user usually has a destination in mind, we model this behavior by choosing one particular node (or cell) in the random graph as the destination. Whenever the mobile 
(a)

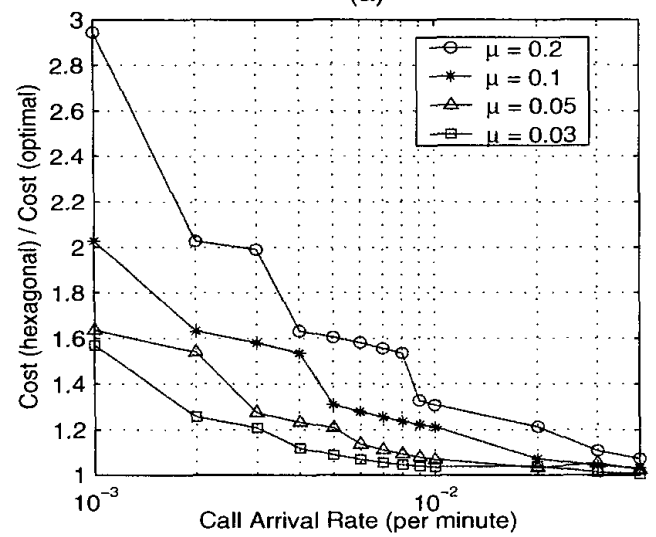

(b)

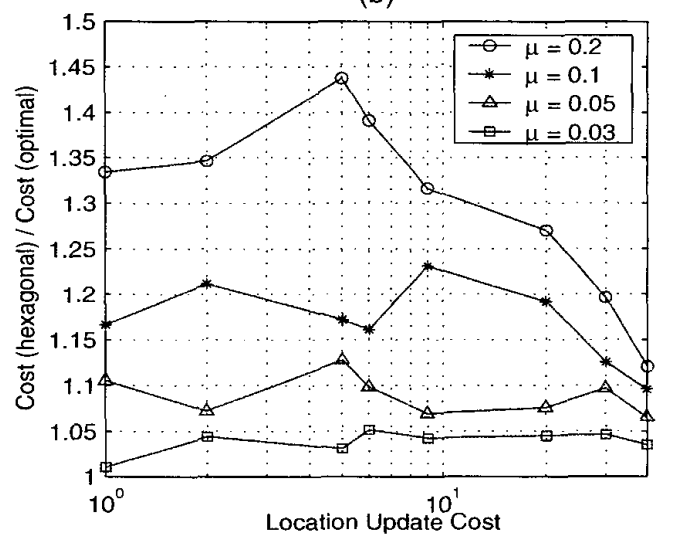

Figure 3 (a) Cost ratio versus call arrival rate $\lambda$ under different cell crossing rate $\mu$; (b) Cost ratio versus location update cost $C_{L U}$ under different cell crossing rate $\mu$.

user leaves the current cell, it moves to a neighboring cell which is closest to the destination. This captures the behavior of moving towards the destination. If the mobile user is staying within the destination cell, after a certain period of time it will move to one of the neighboring cells. This continues until the next call arrives.

We now describe the procedures of comparing the distance thresholds determined from our model to those derived from a hexagonal cell configuration. The goal is to show that the proposed model gives a more accurate update boundary in real wireless cellular environments compared with that derived from a hexagonal cell configuration with random walk movement pattern.

Given the cost and mobility parameters, we first use a hexagonal cell configuration with symmetric random walk movement pattern to obtain the optimal distance threshold. This optimal distance threshold is then applied to the random graph model with Markovian movement pattern. The expected total cost of location update and paging between call arrivals is then determined. This cost is denoted as "Cost (hexagonal)". The term "hexagonal" is used to remind us that the optimal distance threshold is derived from the hexagonal cell configuration. We also use the random graph model with the above movement pattern to determine the minimum expected total cost by solving the optimality equations. This cost is denoted as "Cost (optimal)". The term "optimal" is used to remind us that the update boundary corresponds to the optimal policy. The performance gain is the cost ratio which is defined as Cost (hexagonal) / Cost (optimal).

Unless stated otherwise, the parameters that we use in the following subsections are: call arrival rate $\lambda=0.01$ per minute, the location update cost $C_{L U}=10$, and the paging cost per cell $C_{P}=1$. We assume that the cell residence time follows an i.i.d. Gamma distribution with average time $1 / \mu$. For the value iteration algorithm, we choose $\varepsilon=10^{-}$.

$B$ Results

Figures 4 (a) and (b) show the cost ratio versus the call arrival rate $\lambda$ and the location update cost $C_{L U}$ under different cell crossing rate $\mu$ (per minute). Note that the inverse (or reciprocal) of the average cell residence time is the cell crossing rate. From these figures, we observe that the cost ratio increases when $\mu$ increases or $\lambda$ decreases. And it approaches unity when $\lambda$ or $C_{L U}$ are large. In Figure 3 (a), when the average time between call arrivals is large (i.e., $\lambda$ is small), the optimal update boundary obtained from our model gives a lower cost than the distance threshold derived from the hexagonal model. However, when the average time between call arrivals is small (i.e., $\lambda$ is large), the mobile user does not travel much before a call arrives. Thus, the distance thresholds derive from both methods give the same performance. In Figure 3 (b), the variation of the cost ratio with respect to $C_{L U}$ is due to the changes of the optimal update boundary or distance threshold for different update cost values. When $C_{L U}$ is large, there is no incentive to perform location update. The expected total cost only consists of the paging cost. Thus, the cost ratio approaches unity.

These results imply that in real wireless cellular networks environments where the cell topology is not structured and the user movement pattern is not random, our model can provide a more accurate update boundary than that derived from a hexagonal cell configuration with random walk movement pattern. And by using a more accurate update boundary, the network can maintain a better balance between the processing incurred due to location update and the radio bandwidth utilized for paging between call arrivals.

\section{Sensitivity Analysis}

In order to determine the minimum expected total cost and the update boundary, the optimal policy needs to be calculated. The optimal policy is a function of $\lambda, \mu$, and other cost parameters. Although the cost parameters can be determined by the network, the values of $\lambda$ and $\mu$ may not always be estimated correctly. If that is the case, the policy may not indeed be optimal. We are interested in determining the percentage change of the expected total cost to the variation of the average time between call arrivals and the average cell residence time. The procedures for the sensitivity analysis consist of the following steps: Given the actual call arrival rate $\lambda$ and other cost and mobility parameters, we first determine the minimum expected total cost, denoted as Cost (optimal). Let $\hat{\lambda}$ denote the estimated call arrival rate and $\Delta_{\lambda}$ denote the percentage change of the average time between call arrivals. These parameters are related by the following equation:

$$
\hat{\lambda}^{-1}=\left(1+\Delta_{\lambda}\right) \lambda^{-1} \text {. }
$$


(a)

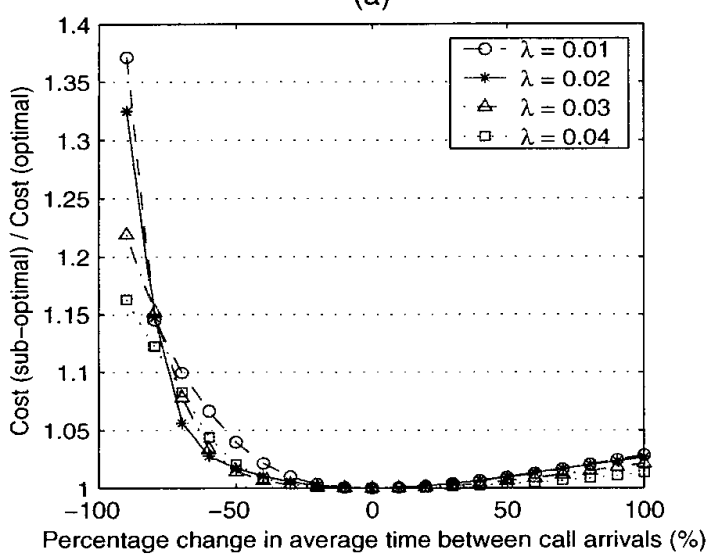

(b)

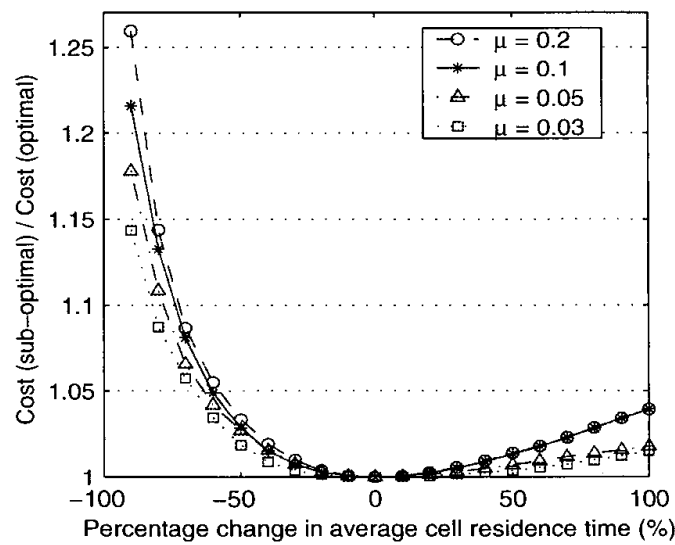

Figure 4 (a) Cost ratio versus $\Delta_{\lambda}$ under different call arrival rate $\lambda$; (b) Cost ratio versus $\Delta_{\mu}$ under different cell crossing rate $\mu$.

Based on the estimated call arrival rate $\hat{\lambda}$ and other parameters, the sub-optimal policy is determined. From this suboptimal policy and other cost and mobility parameters (i.e., $\lambda$, $\mu$, etc), the sub-optimal expected total cost, denoted as Cost (sub-optimal), is computed. The change of the expected total cost with respect to the variation of the average time between call arrivals is characterized by the cost ratio, which is defined as: Cost (sub-optimal) / Cost (optimal).

We also use the similar procedures describe above to investigate the change of the expected cost to the variation of the average cell residence time. Figure 4 (a) shows the cost ratio versus $\Delta_{\lambda}$ under different call arrival rate $\lambda$ and Figure 4 (b) shows the cost ratio versus $\Delta_{\mu}$ under different cell crossing rate $\mu$. From these figures, we observe that the cost ratio is more sensitive to the under-estimation of both $\lambda$ and $\mu$. If the target cost ratio has to be less than 1.05 (i.e., $5 \%$ difference between the optimal and sub-optimal cost), then $\Delta_{\lambda}$ has to be greater than $-50 \%$ and $\Delta_{\mu}$ has to be greater than $-60 \%$. These results imply that if there is uncertainty in estimating $\lambda$ or $\mu$, it may be better to over-estimate the values in order to reduce the cost ratio difference.

\section{CONCLUSIONS}

In this paper, we proposed a stochastic model to analyze the distance-based location update algorithm. Numerical results indicate that the proposed model gives a more accurate update boundary in real wireless cellular environment than that derived from a hexagonal cell configuration with random walk movement pattern. And by using a more accurate location update boundary, the network can maintain a better balance between the processing incurred due to location update and the radio bandwidth utilized for paging between call arrivals. Results from the sensitivity analysis show that if there is uncertainty in estimating the call arrival rate or cell residence time, it may be better to over-estimate the values in order to reduce the cost ratio difference.

\section{REFERENCES}

[1] V. Wong and V. Leung, "Location management for next generation personal communication networks," IEEE Network, vol. 14, no. 5, Sept./Oct. 2000.

[2] U. Madhow, M. Honig, and K. Steiglitz, "Optimization of wireless resources for personal communications mobility tracking," IEEE/ACM Trans. Networking, vol. 3, no. 4, pp. 698-707, Dec. 1995.

[3] J. Ho and I. F. Akyildiz, "Mobile user location update and paging under delay constraints," ACM/Baltzer J. Wireless Networks, vol. 1, no. 4, pp. 413-425, Dec. 1995.

[4] C. Rose, "Minimizing the average cost of paging and registration: A timer-based method," ACM/Baltzer $J$. Wireless Networks, vol. 2, no. 2, pp. 109-116, June 1996.

[5] I. F. Akyildiz, J. Ho, and Y.-B. Lin, "Movement-based location update and selective paging for PCS networks," IEEE/ACM Trans. Networking, vol. 4, no. 4, pp. 629-638, Aug. 1996.

[6] B. Liang and Z. Haas, "Predictive distance-based mobility management for PCS networks," in Proc. IEEE INFOCOM'99, New York, NY, March 1999.

[7] C. Rose, "State-based paging/registration: A greedy technique," IEEE Trans. Vehicular Technology, vol. 48, no. 1, Jan. 1999.

[8] A. Bhattacharya and S. K. Das, "LeZi-Update: An information-theoretic approach to track mobile users in PCS networks," in Proc. ACM/IEEE MobiCom'99, Seattle, WA, Aug. 1999.

[9] W. Wang and I. F. Akyildiz, "Intersystem location update and paging schemes for multitier wireless networks, in Proc. ACM MobiCom'00, Boston, August 2000.

[10] S. K. Sen, A. Bhattacharya, and S. K. Das, "A selective location update strategy for PCS users," ACM/Baltzer J. Wireless Networks, vol. 5, no. 5, pp. 313-326, Sept. 1999.

[11] M. L. Puterman, Markov Decision Processes: Discrete Stochastic Dynamic Programming, John Wiley \& Sons Inc., 1994.

[12] W.-S. V. Wong, "Stochastic control of inter-switch handoff and location update in wireless cellular networks," Ph.D.'s Thesis, The University of British Columbia, Canada, April 2000.

[13] M. Zonoozi and P. Dassanayake, "User mobility modeling and characterization of mobility patterns," IEEE J. Select. Areas Commun., vol. 15, no. 7, pp. 1239-1252, September 1997.

[14] E. W. Zegura, K. L. Calvert, and M. J. Donahoo, "A quantitative comparison of graph-based models for Internet topology," IEEE/ACM Trans. Networking, vol. 5, no. 6, Dec. 1997. 